\title{
Practical Considerations of ASK Modulated Passive Tags
}

\author{
Antonis G. Dimitriou ${ }^{1}$, Aggelos Bletsas ${ }^{2}$, John N. Sahalos ${ }^{3}$ \\ ${ }^{1}$ Dept. of Electrical \& Computer Engineering, Aristotle University of Thessaloniki, Thessaloniki, Greece, antodimi@auth.gr \\ ${ }^{2}$ Dept. of Electronic \& Computer Engineering, Technical University of Crete, Chania, Greece, aggelos@telecom.tuc.gr \\ ${ }^{3}$ Dept. of Electrical \& Computer Engineering, University of Nicosia, Nicosia, Cyprus, sahalos.j@unic.ac.cy
}

\begin{abstract}
This paper investigates practical, wideband considerations that affect the performance of modulated passive UHF RFID tags. The tag-antenna's structural scattering term can be favorably exploited increasing the total backscattered power, by properly selecting the circuit's parameters within realistic values, as shown herein. Frequency domain analysis of the simplified circuit is carried out in the operational UHF frequency band. Small performance-degradation, with respect to power at the rectifier and mean backscattered power, is recorded away from resonance. However, Bit Error Rate is shown to vary greatly within the frequency band.
\end{abstract}

Keywords; RFID; ASK; passive tags; sensitivity, wideband analysis

\section{INTRODUCTION}

In UHF RFID communications a tag modulates a continuous wave $(\mathrm{CW})$ signal transmitted by the reader, by changing its load between two states, thus changing the phase or/and the magnitude of the backscattered signal that arrives at the reader [1]. Performance of the system depends on proper load selection for the two states of the tag, so as to ensure enough backscattered power at the reader and sufficient separation in the complex plane of the backscattered fields that correspond to the two states of tag-loading.

In addition, for passive tags, where no battery source exists, their circuits are driven from the transmitted power of the reader. The ability of the tag to "wake up" and store this power represents the main limiting factor for the identification range of high frequency RFID systems [1]. As a result, it is of critical importance to draw as much of the incident power as possible. Rectification of the incident power in the tag's circuitry has been thoroughly analyzed [1]. Another equally important parameter in the tag's performance is the proper tag-antenna design [2]. As shown in [2], the tag's antenna should be inductive in the operating frequency bandwidth, in order to conjugate-match the capacitance of the rectifier. A third parameter, representing the particularity of backscattering communications as opposed to classical one-way communication, is the generation of the "structural scattering term", [3], [4], by the tag's antenna. It has been shown (e.g. [5]) that the current on the Thévenin's equivalent of an antenna connected to a load, does not quantify the field reradiated by the antenna (with the exception of specific antennas). Therefore, a specific term in the backscattered field has been introduced that is independent of antenna-loading; hence the term "structural", as it depends only on the structure of the antenna. The "structural scattering term" shapes the modulated backscattered signal, and represents a critical parameter for proper tag design. Antenna "structural mode" has been overlooked in the tag-design process until recently [6].

Extending prior-work on the field [6], [7], we elucidate the importance of the tag-antenna's structural term in the front-end of the reader's receiver and carry out a frequency-domainanalysis of the proposed simplified tag-design, based on realistic values from commercially available tag ICs.

\section{ANTENNA BACKSCATTERING}

The current at the Thévenin's equivalent circuit of an antenna connected to a load does not quantify its backscattered field. To properly evaluate the backscattered field, we should calculate a "modified" tag's radar cross section $\sigma$, derived in [4]:

$$
\begin{gathered}
\sigma=\frac{\lambda^{2}}{4 \pi} G_{t a g}^{2}\left|\Gamma-A_{s}\right|^{2} \\
\Gamma=\frac{Z_{L}-Z_{a}^{*}}{Z_{L}+Z_{a}},
\end{gathered}
$$

$\lambda$ is the wavelength at the operating frequency, $G_{t a g}$ is the tag's antenna gain and $A_{s}$ is a complex parameter independent of the load that quantifies the structural mode term of the antenna. $\Gamma$ expresses a modified reflection coefficient, defined in (2), where $Z_{L}, Z_{a}$ is the load's and the antenna's impedance respectively.

Equation (1) is valid for a monostatic reader-antenna case and assumes no polarization mismatch. The analytical expression for a bistatic configuration with polarization mismatch can be found in [4] and does not affect the tag-design principles that will be presented in the following sections.

The term $A_{s}$ can be measured or simulated for any tag's antenna by evaluating or measuring the radar cross section for three different loads as demonstrated in [6]. Measurement examples of $A_{s}$ for tag antennas can be found in [3], [8] and [9]. 
$A_{s}$ is usually omitted in the literature for the tag. That is coming from the claim that this term doesn't influence Bit Error Rate (BER) performance of the system, since it is common in the backscattered field, regardless of the connected load. Even though this claim is correct, $A_{s}$ strongly influences the total backscattered carrier power [6]; thus, the structural mode should be also taken into account.

\section{ANALYSIS OF TAG'S EQUIVALENT CIRCUIT}

\section{A. Design Considerations}

Let $\Gamma_{i} i=1,2$ denote the reflection coefficient for each tag's load state, given in (2) by properly setting $Z_{L}$. It was shown in [6] that proper tag design should carefully consider 2 constraints:

1) maximization of average backscattered power, expressed as:

$$
\max \left\{\sigma_{1}+\sigma_{2}\right\}
$$

where $\sigma_{i}$ is given in (1), by replacing $\Gamma$ with $\Gamma_{i}$, and 2) minimization of the BER at the reader expressed as:

$$
\max \left\{\left|\Gamma_{1}-\Gamma_{2}\right|\right\}
$$

Constraints (3) and (4) are orthogonal. Constraint (4) is critical for the performance of the reader's detector and has been considered in the related literature (e.g. [10]). Constraint (3) is critical for the performance of the reader's front end and has been overlooked until recently [6]. The reader's antenna receives the wanted (typically small) modulated (by the tag information) backscattered signal together with strong (typically stronger) carrier reflections from the surrounding environment (e.g. due to reflections from walls or other surfaces in the vicinity of the reader). In the case of monostatic readers, an "unwanted", attenuated fraction of the strong carrier frequency also leaks into the receiver's chain through the circulator (or coupler). This problem (also common in monostatic radars) is partially controlled by implementing a "reflected power canceller" circuit like the one proposed in [11] or a carrier suppression circuit like the one in [12] (where $42 \mathrm{~dB}$ suppression is accomplished).

The difference between these unmodulated signals and the (much smaller) backscattered modulated signal should be kept as small as possible in order to avoid desensitization of the receiver and in order for the system to operate within its spurious free dynamic range (SFDR). This parameter had been ignored, without any "disastrous" reported results. The reason for this "hidden" effect is that early RFID systems, particularly those with passive RFID tags, were forward link limited. The necessary minimum power for a passive tag to "wake up" was large, and as a consequence the average backscattered power was relatively large compared to the sensitivity of the reader's front-end. However, as the minimum threshold at the tag reduces, the reader should be able to cope with very small modulated signals (in the order of $-80 \mathrm{dBm}$ to $-90 \mathrm{dBm}$ ), as commented in [13], [14].
By substituting (1) in (4), we find that BER minimization does not require knowledge of the structural mode term As. However, by substituting (1) in (3), it is found that to maximize average backscattered power, knowledge of $A_{s}$ is essential.

\section{1) Passive Tags}

In the case of passive tags, maximum power transfer should be maintained at the sleeping state of the tag. Hence, an additional constraint is [7]:

$$
\min \left\{\left|\Gamma_{1}\right|\right\} \Rightarrow \max \text { power transfer }
$$

\section{B. Design Criteria}

For optimal performance of passive RFID tags, we have from (5):

$$
\left|\Gamma_{1}\right|=0
$$

Then, by substituting (6) in (3) and using (2), we get:

$$
\begin{gathered}
\max \left\{\left|\Gamma_{1}-A_{s}\right|+\left|\Gamma_{2}-A_{s}\right|\right\}=\max \left\{\left|A_{s}\right|+\left|\Gamma_{2}-A_{s}\right|\right\} \Rightarrow \\
\left|\Gamma_{2}\right|=1,\left\langle\Gamma_{2}=\left\langle-A_{s}, \max \left\{\left|A_{s}\right|\right\}\right.\right.
\end{gathered}
$$

Equation (8) states that the selection of load at state 2 should be such that the magnitude of the modified reflection coefficient $\Gamma_{2}$ should equal 1 and its phase should equal the phase of $-A_{s}$. Furthermore, backscattered power increases with increasing $\left|A_{s}\right|$. Constraint (4) is also fulfilled, because for $\Gamma_{l}=0$ (6), we should have $\left|\Gamma_{2}\right|=1$ (see (8)). The above design principles are shown in Fig. 1. We start by drawing the common term $-A_{s}$, represented by a vector in the complex plane. Then, we draw a circle of unit-radius centered at $-A_{s}$, representing the boundary of maximum $\left|\Gamma_{i}\right|$. The surface enclosed by that circle represents the area, where $\Gamma_{1}-A_{s}$ and $\Gamma_{2}-A_{s}$ could belong. After selecting the desired values for $\Gamma_{i}$, we can directly determine the appropriate impedance of the loads from the normalized (with respect to the antenna's resistance $R_{a}$ ) Smith chart, shown "faded" in Fig. 1.

An example of the effect of the structural term combined with proper selection for $\Gamma_{2}$ is shown in Fig. 2. We assume $\left|A_{s}\right|=1,<A_{s}=45^{\circ}, \Gamma_{I}=0,\left|\Gamma_{2}\right|=1$ (constraint (4) is valid), and we raise the phase of $\Gamma_{2}$ from $0^{\circ}$ to $360^{\circ}$. Normalized backscattered power is drawn in polar plot (in $\mathrm{dBs}$ ) with respect to its maximum value accomplished for $<\Gamma_{2}=-<A_{s}$; an improvement of $7 \mathrm{dBs}$ is demonstrated, by proper selection of load at state 2 .

\section{Tag's Equivalent Circuit}

The tag's equivalent circuit is demonstrated in Fig. 3. With respect to prior art [7], the antenna has been properly modeled, including the inductance, in order to carry out a frequency analysis of the circuit. The following design parameters are considered: 
$a=R_{i} / R_{a}, b=X_{a} / X_{i}, d=X / X_{i}, Q_{i}=X_{i} / R_{i}$

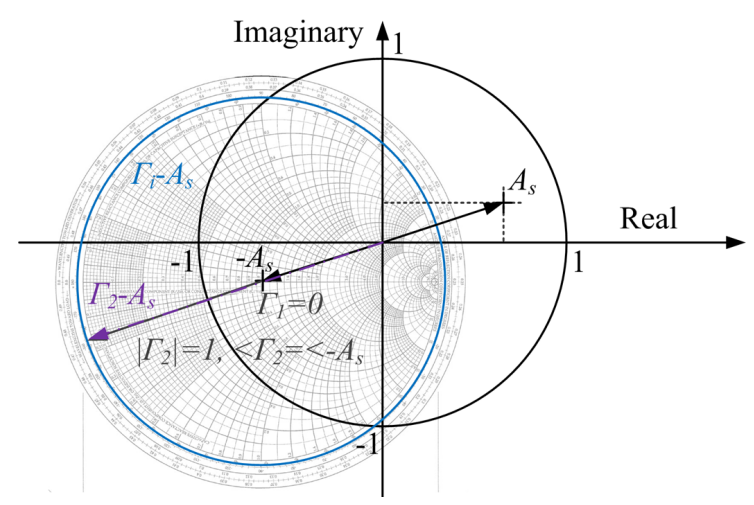

Figure 1. Optimum load selection for passive tag and ASK modulation.

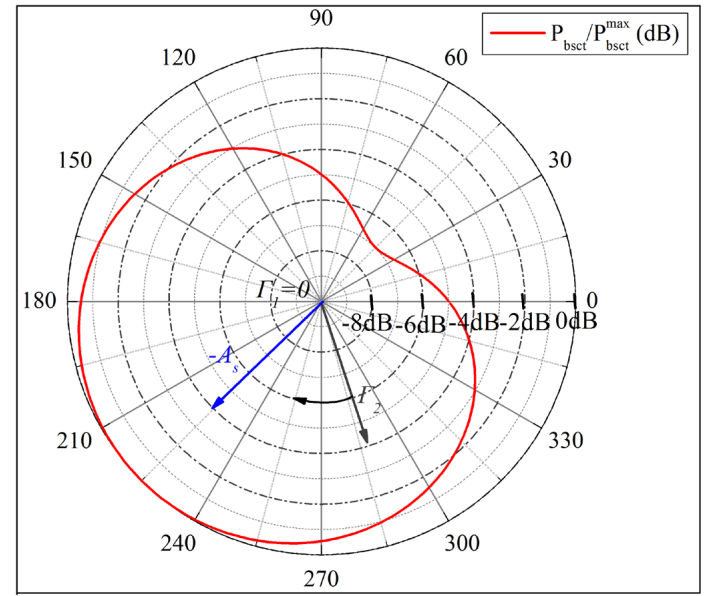

Figure 2. Backscattered power for varying $\Gamma_{2}$ and $\left|\Gamma_{2}\right|=1$.

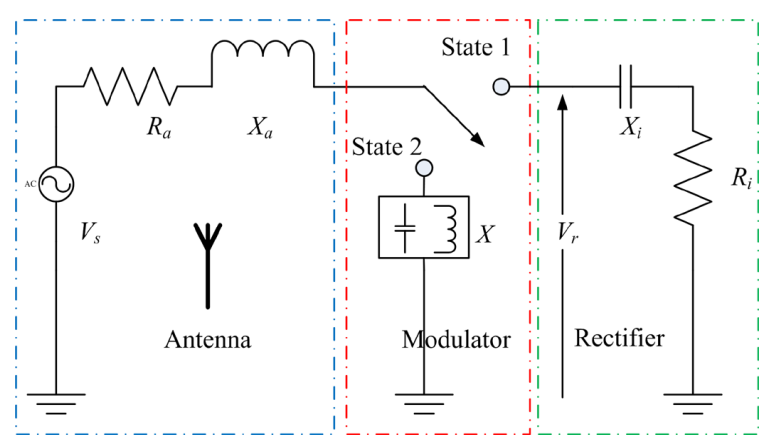

Figure 3. Tag's simplified equivalent circuit.

By analysis of the circuit of Fig. 3, we have at state 1 the voltage $V_{r}$ at the rectifier in the following form:

$$
\frac{V_{r}}{V_{s}}=\frac{a\left(1-j Q_{i}\right)}{1+a+j a Q_{i}(b-1)}
$$

where $V_{s}$ represents the equivalent voltage source resulting from the incident field at the antenna. The reflection coefficient at states 1 and 2 are respectively:

$$
\begin{gathered}
\Gamma_{1}=\frac{(a-1)+j a Q_{i}(b-1)}{(a+1)+j a Q_{i}(b-1)} \\
\Gamma_{2}=\frac{-1+j a Q_{i}(b+d)}{1+j a Q_{i}(b+d)}
\end{gathered}
$$

We represent the complex tag's antenna structural mode as $A_{s}=A_{s r}+\mathrm{j} A_{s i}$. From (6) and (11), we get:

$$
a=1, b=1
$$

From (8), (12) and (13), we have:

$$
d=-1+\frac{A_{s r}-\left|A_{s}\right|}{A_{s i} Q_{i}}
$$

From (10), we find that the voltage at the input of the rectifier increases with $Q_{i}$. However, $Q_{i}$ represents the quality factor of the $R_{i}-X_{a}-X_{i}$ series circuit at resonance and is connected to the desired operating bandwidth $\Delta f$ of the tag and the carrier frequency $f_{0}$ by the following equation: $Q_{i}=f_{0} / \Delta f$. For a tag to be operational globally in the UHF RFID band $(858 \mathrm{MHz}-$ $930 \mathrm{MHz}), Q_{i}$ should be smaller than 12.4 [7]. Input impedance values of commercially available UHF band RFID chips, [15] - [17], are summarized in Table I. The chip's impedance is typically described in the datasheet of the manufacturer by a resistor $R_{\|}$connected in parallel to a capacitor $C_{\|}$. Their series equivalent values for the frequency of $896.5 \mathrm{MHz}$, corresponding to the circuit of Fig. 3, are also given in Table 1, as well as the resultant $Q_{i}$. Notice that $Q_{i}$ is smaller than 12.4 for the three chips, which represents the marginal value for global operation of a UHF tag. $R_{i}$ ranges from $15.7 \Omega$ to $28.5 \Omega, X_{i}$ from $145 \Omega$ to $204.8 \Omega$, and $Q_{i}$ ranges from 7.18 to 9.2. In all cases the parallel capacitor is in the order of $1 \mathrm{pF}$ and the parallel resistor in the order of $1.5 \mathrm{~K} \Omega$.

TABLE I. CAPACITANCE \& RESISTANCE OF COMMERCIAL CHIPS

\begin{tabular}{|c|c|c|c|c|c|c|}
\hline Chip & $\boldsymbol{C}_{\|}(\boldsymbol{p} \boldsymbol{F})$ & $\boldsymbol{R}_{\|}(\boldsymbol{K} \boldsymbol{\Omega})$ & $\boldsymbol{X}_{\boldsymbol{i}}$ & $\boldsymbol{R}_{\boldsymbol{i}}$ & $\boldsymbol{Q}_{\boldsymbol{i}}$ & $\mathbf{1} \boldsymbol{\omega}_{\boldsymbol{0}} \boldsymbol{C}_{\|}$ \\
\hline $\begin{array}{c}\text { Alien Higgs 3 } \\
\text { [15] }\end{array}$ & 0.85 & 1.5 & 204.8 & 28.5 & 7.18 & 208 \\
\hline $\begin{array}{c}\text { NXP UCODE } \\
\text { G2XM \& } \\
\text { G2XL [16] }\end{array}$ & 0.9 & 1.768 & 194.8 & 21.7 & 8.96 & 197 \\
\hline $\begin{array}{c}\text { Impinj Monza } \\
\text { 3 [17] }\end{array}$ & 1.21 & 1.35 & 145 & 15.7 & 9.2 & 146 \\
\hline
\end{tabular}

IV. WIDEBAND PERFORMANCE ANALYSIS

Performance of the circuit in terms of backscattered power, voltage at the rectifier and range can be found in our earlier work [7]. In this section, we will carry out a frequency domain analysis of the circuit, in order to evaluate "global" performance of the proposed tag.

We assume that the tag is destined to operate at the UHF band $865 \mathrm{MHz}-928 \mathrm{MHz}$. The parameters of the circuit are 
optimized for the center frequency $f_{0}=896.5 \mathrm{MHz}$, where we assume: $a_{f_{0}}=1, b_{f_{0}}=1, d_{f_{0}}$ given by (14), and $Q_{f_{0}}$ depending on the tag-chip's impedance, where the subscript $f_{0}$ denotes the value of the corresponding variable at the center frequency.

The series equivalent frequency-dependent $R_{i}(f), X_{i}(f)$ values of the circuit are related to the parallel values by the following equations:

$$
\begin{gathered}
R_{i}(f)-j X_{i}(f)=\frac{R_{\|}}{1+\left(2 \pi f R_{\|} C_{\|}\right)^{2}}-j \frac{2 \pi f R_{\|}^{2} C_{\|}}{1+\left(2 \pi f R_{\|} C_{\|}\right)^{2}}= \\
=\frac{R_{\|}}{1+k^{2} Q_{f_{0}}^{2}}-j \frac{R_{\|} k Q_{f_{0}}}{1+k^{2} Q_{f_{0}}^{2}}=R_{i}(k)-j X_{i}(k) \\
Q_{i}(f)=2 \pi f R_{\|} C_{\|}=2 \pi k f_{0} R_{\|} C_{\|}=k Q_{f_{0}}=Q_{i}(k)
\end{gathered}
$$

$$
\text { and } k=f / f_{0}
$$

The real part of the tag's antenna $R_{a}$ is expected to increase with frequency $f$, because of the resultant increase of its electrical length [2], [9]. We can approximate this variation linearly $R_{a}(f)=R_{a}\left(f_{0}\right)+\lambda\left(f-f_{0}\right)$, where $\lambda$ is the appropriate slope. In [9], $\lambda=0.1$ and $f_{0}$ should be replaced in MHz. In [2], $\lambda=0.4$. Similarly the imaginary part of the antenna $X_{a}(f)$ is also expected to increase proportionally to $2 \pi f L$ [2]. Hence the frequency variations of $a(f), b(f)$ are given by:

$$
\begin{gathered}
a(f)=\frac{R_{i}(f)}{R_{a}(f)}=\frac{\left[R_{\|} /\left[1+\left(2 \pi f R_{\|} C_{\|}\right)^{2}\right]\right]}{R_{a}\left(f_{0}\right)+\lambda\left(f-f_{0}\right)}= \\
=a_{f_{0}} \frac{R_{\|}\left(1+Q_{f_{0}}^{2}\right)}{\left(1+k^{2} Q_{f_{0}}^{2}\right)\left[R_{\|}+a_{f_{0}} \lambda f_{0}(k-1)\left(1+Q_{f_{0}}^{2}\right)\right]}=a(k) \\
b(f)=\frac{X_{a}(f)}{X_{i}(f)}=\frac{2 \pi k f_{0} L}{2 \pi k f_{0} R_{\|}^{2} C_{\|} /\left[1+\left(2 \pi k f_{0} R_{\|} C_{\|}\right)^{2}\right]}=b(k)
\end{gathered}
$$

For the typical values of the chip's impedance, $R_{\|} \sim 1.5 K \Omega, C_{\|} \sim 1 p F$, we have $\left(2 \pi f R_{\|} C_{\|}\right)^{2} \gg 1$ and therefore $X_{i} \approx 1 /\left(2 \pi f C_{\|}\right)$(the approximated values for the three chips are given in the last column of Table I). In such case (19) is simplified to:

$$
b(f)=\frac{X_{a}(f)}{X_{i}(f)}=\frac{2 \pi k f_{0} L}{1 / 2 \pi k f_{0} C_{\|}}=k^{2} b_{f_{0}}
$$

Similarly, for typical values of chip's impedance, we can write for the capacitance $X$ that affects parameter $d$ :

$$
d(f)=\frac{1 / 2 \pi f C}{1 / 2 \pi f C_{\|}}=\frac{1 / 2 \pi k f_{0} C}{1 / 2 \pi k f_{0} C_{\|}}=d_{f_{0}}
$$

Hence, $d$ can be considered constant in the entire frequency band.

For $f_{0}=896.5 \mathrm{MHz}$ and the frequency varying from $865 \mathrm{MHz}$ to $928 \mathrm{MHz}, k$ increases from 0.964 to 1.035 . We consider a tag with $A_{s}=0.6047+\mathrm{j} 0.5042$ and the "Alien Higgs" chip with $Q_{f_{0}}=7.18$. The antenna is power matched to the chip's impedance at $f_{0}$. From (14) we get $d_{f_{0}}=-1.05$. By substituting (16), (18), (20) and (21) in (11) and (12), we calculate the vectors $\Gamma_{I}(f)-A_{s}$ and $\Gamma_{2}(f)-A_{s}$ for three values of $\lambda=\{0,0.1,0.4\}$. We evaluate the tag's performance in terms of three parameters: $a$ ) power at the rectifier vs the maximum power transfer at resonance $f_{0}$ in $(\mathrm{dB}): 10 \log 10\left[P_{\text {rect }}(f) / P_{\text {rect }}\left(f_{0}\right)\right]$ (Fig. 4$\left.), b\right)$ Backscattered power at the reader vs its value at the center frequency $f_{0}$ in dB: $10 \log 10\left[P_{b s c t}(f) / P_{b s c t}\left(f_{0}\right)\right]$ (Fig. 5), and $c$ ) BER performance (Fig. 6) given by:

$$
B E R=Q\left(\sqrt{|A| / 2 N_{0}}\left|\Gamma_{1}(f)-\Gamma_{2}(f)\right|\right)
$$

where $Q(x)=(1 / \sqrt{2 \pi}) \int_{x}^{\infty} e^{\left(-x^{2} / 2\right)} d x, A$ is a common term in the backscattered field in both states of the tag's load and accounts for the channel losses [10] and $N_{0}$ is the expected power of the noise level at the reader, which is considered as complex zero mean circularly symmetric Gaussian random variable.

The results of Figs. 4, 5 indicate that acceptable performance is maintained even at the margins of the frequency band. Worst case analysis shows that power at the tag decreases by $0.85 \mathrm{~dB}(\lambda=0.4, f=0.865 \mathrm{GHz})$, which results in approximately $9 \%$ range reduction, assuming power $P$ decreases at an inverse square law with distance $\left(P \sim 1 / r^{2}\right)$. Backscattered power is reduced by $1.65 \mathrm{dBs}$ at most. However, Fig. 6 shows that BER performance of the system is very sensitive to the differences $|\Delta \Gamma(f)|=\left|\Gamma_{l}(f)-\Gamma_{2}(f)\right| . \Delta \Gamma$ changes from 0.71 to 1.0 within the operational frequency band. BER for these two marginal values is given in Fig. 6. It is found that for a given signal to noise ratio, the BER performance could vary greatly in the frequency band.

\section{CONCLUSIONS}

This work extends prior art in the field of passive RFID tag design. It is shown that the antenna structural term should be favorably exploited to increase mean backscattered power, thus improving the performance at the front-end of the reader's receiver. Furthermore, wideband analysis of the tag shows that good performance in terms of power at the rectifier and backscattered power is maintained in the entire RFID UHF frequency band. However, BER may increase significantly away from the resonant frequency. 


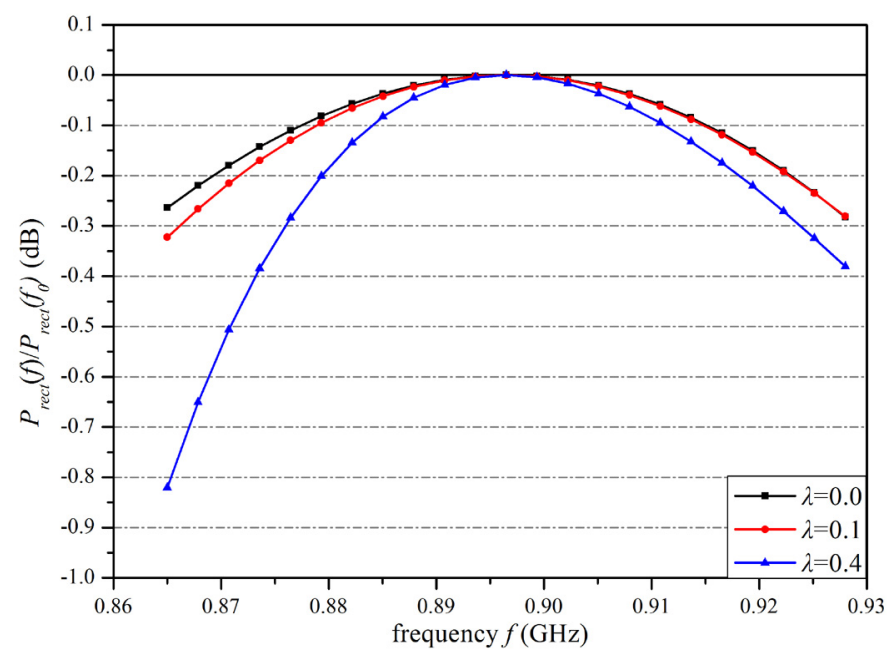

Figure 4. Power transfer to the rectifier vs. frequency.

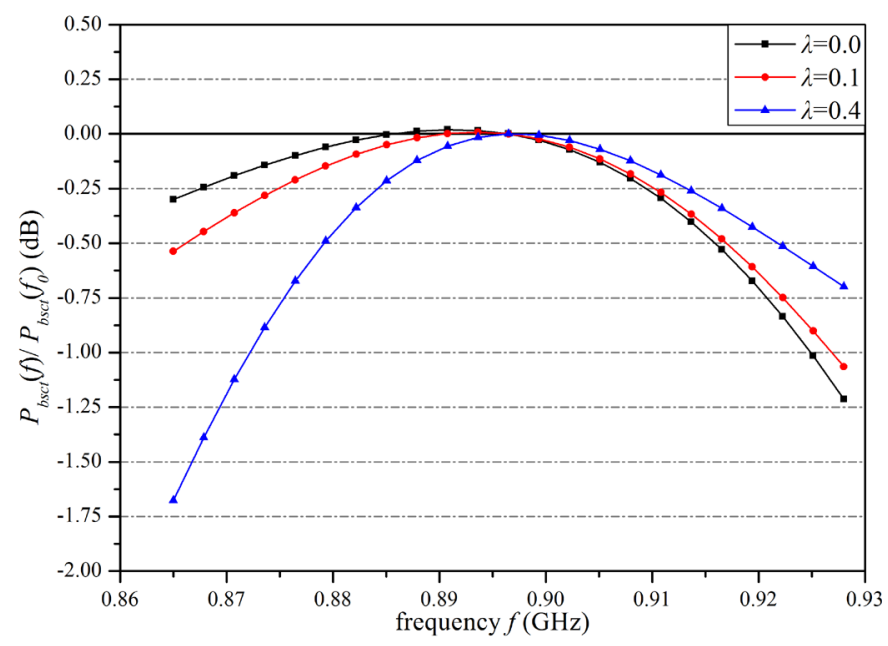

Figure 5. Backscattered power vs frequency.

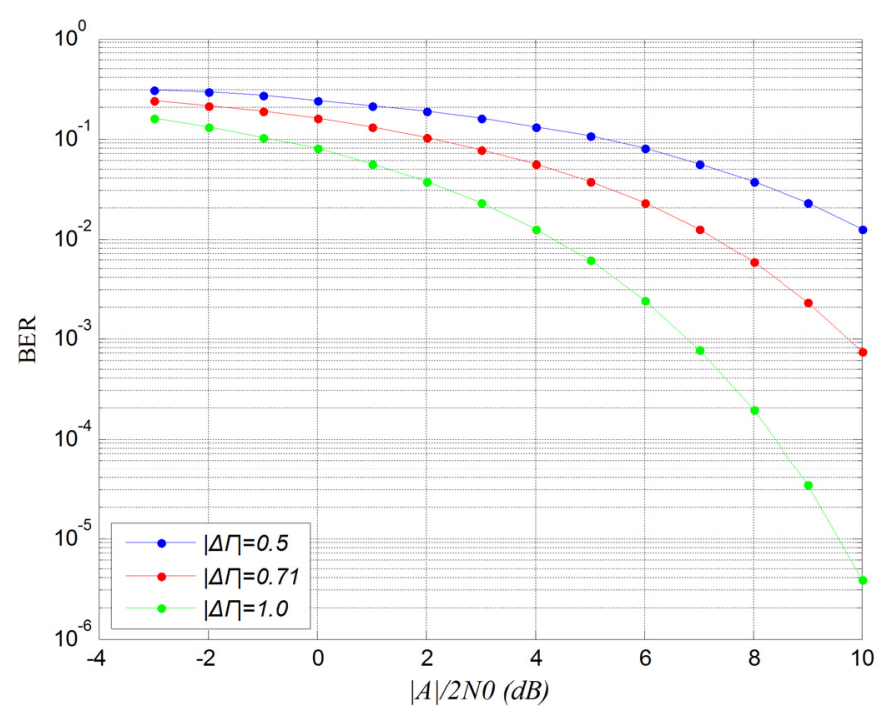

Figure 6. BER performance for the marginal cases of expected $\Delta \Gamma$.

\section{ACKNOWLEDGMENT}

This research was co-funded by the Greek Ministry of Education and the E.U./European Social Fund, Operational Program "Education and Life-Long Learning (EDULLL)", call "Thales", project short-name "RFID-CORE".

\section{REFERENCES}

[1] D. M. Dobkin, The RF in RFID, Passive UHF RFID in Practice, Newnes, Elsevier, 2008.

[2] A.H. Rida, Li Yang; S. Serkan Basat, A. Ferrer-Vidal, S. Nikolaou, M. M. Tentzeris, "Design, development and integration of novel antennas for miniaturized UHF RFID tags," IEEE Trans. Antennas Propagat., vol. 57 , no 11 , pp. $3450-3457$.

[3] C. A. Balanis, Antenna Theory Analysis and Design, Third Edition, New Jersey, John Wiley \& Sons, pp. 98-104, 2005.

[4] R. B. Green, The General Theory of Antenna Scattering, Dissertation, Ohio State University, 1963.

[5] R. W. P. King and C. W. Jr. Harrison, "The receiving antenna," Proc. I.R.E., no 21, January, 1944

[6] A. Bletsas, A. G. Dimitriou, J. N. Sahalos, "Improving backscatter radio tag efficiency," IEEE Trans. Microwave Theory \& Techniques, vol. 58, no 6, pp. 1502-1509, June 2010.

[7] A. G. Dimitriou, A. Bletsas and J. N. Sahalos, "On the design of passive RFID tags for ASK modulation," 5th European Conference on Antennas \& Propagation (EuCAP), Rome, 11-15 April, 2011.

[8] H.-Y. Chen, A. S. Bhadkamkar, T.-H. Chou, D. W. van der Weide, "Vector backscattered signals improve piggyback modulation for sensing with passive UHF RFID tags," IEEE Trans. On Microwave Theory and Techniques, vol 59, no 12, pp. 3538 - 3545, Dec. 2011.

[9] C.-C. Yen, A. E. Gutierrez, D. Veeramani, and D. van der Weide, "Radar cross-section analysis of backscattering RFID tags," IEEE Antennas Wireless Propag. Lett., vol. 6, pp. 279-281, 2007.

[10] F. Fuschini, C. Piersanti, F. Paolazzi, G. Falciasecca, "Analytical approach to the backscattering from UHF RFID transponder," IEEE Antennas \& Wireless Propagation Letters, vol. 7, pp. 33-35, 2008.

[11] P. Pursula, M. Kiviranta, and H. Seppä, "UHF RFID reader with reflected power canceller," IEEE Microwave and Wireless Components Letters, vol. 19, no. 1, pp. 48-50, Jan. 2009.

[12] T. Brauner and X. Zhao, "A novel carrier suppression method for RFID," IEEE Microwave and Wirless Components Letters, vol. 19, no. 3, pp. 128-130, March 2009.

[13] A. G. Dimitriou, A. Bletsas, and J. N. Sahalos, "Room coverage improvements of UHF RFID with commodity hardware," IEEE Antennas and Propagation Magazine, vol. 53, no. 1, February 2011.

[14] R. Chakraborty, S. Roy, and V. Jandhyala,"Revisiting RFID link budgets for technology scaling: range maximization of RFID tags," IEEE Trans. Microwave Theory and Techniques, vol. 59, no. 2, pp. 496503, Feb. 2011.

[15] "Higgs-3 data sheet", Alien, Morgan Hill, California, USA.

[16] "UCODE G2XM and UCODE G2XL data sheet," NXP, Eindhoven, The Netherlands.

[17] “IPJ-P5002-D2 data sheet," Impinj, Seattle, Washington USA. 